\title{
CW Ultraviolet and Visible Laser Action from Ionized Silver in an Electron Beam Generated Plasma
}

\author{
B. WERNSMAN, MEMBER, IEEE, J. J. ROCCA, MEMBER, IEEE, AND H. L. MANCINI
}

\begin{abstract}
Continuous wave laser oscillation was obtained in the 318.1 $\mathrm{nm}$ and the $478.8 \mathrm{~nm}$ lines of AgIl in a neon-silver vapor mixture excited by glow discharge electron beams. Laser output powers of 14 and $60 \mathrm{~mW}$ were obtained in the ultraviolet and blue transitions, respectively, using nonoptimized optical cavities.
\end{abstract}

$P$ REVIOUSLY, we reported laser action in more than 50 ionic and atomic laser lines using electron beam excitation of helium-metal vapor plasmas [1]-[5]. Different population inversion mechanisms including charge transfer [1], [2], collisional electron-ion recombination [3], penning ionization [4], and dissociative excitation [5] have been successfully used to obtain laser oscillation in plasmas generated by glow discharge electron beams. DC electron beam excitation has been demonstrated to produce high $\mathrm{CW}$ laser powers as $1.2 \mathrm{~W}$ was obtained in the blue lines of ZnII [1]. However, up to date $\mathrm{CW}$ laser action by electron beam excitation was limited to the infrared and the visible regions of the spectrum and to the use of helium as the major atomic component of the gain medium. Herein we report the operation of a CW electron beam pumped laser in the ultraviolet for the first time. Also we have demonstrated the use of neon as a buffer gas thereby significantly increasing the number of possible laser lines that might be excited with this type of excitation by means of selective energy transfer processes involving quasi-resonant collisions [6].

$\mathrm{CW}$ laser action was obtained in the $318.1 \mathrm{~nm}\left[5 s^{2}{ }^{1} G_{4}-5 p\right.$ $\left.{ }^{3} F_{3}^{\circ}\right]$ and the $478.8 \mathrm{~nm}\left[5 s^{2}{ }^{1} D_{2}-5 p^{1} P_{1}{ }^{\circ}\right]$ lines of AgII in a neon-silver plasma excited by a multikilovolt dc electron beam. The laser upper levels are populated by charge transfer collisions between ground state neon ions and ground state silver atoms as previously observed to occur in hollow cathode discharges [7]. The majority of the neon ions are created by electron impact ionization as a consequence of the collisions between high-energy electrons and neon atoms.

The laser setup employed in the experiments is similar to that recently used to obtain laser action in the infrared in a HeAg mixture, and it has been described in detail in a previous publication [2]. The laser active medium is a neon-silver

Manuscript received September 11, 1989; revised October 17, 1989. This work was supported by AFOSR Grant 87-0290 and a National Science Foundation Presidential Young Investigator Award to J. J. Rocca.

The authors are with the Department of Electrical Engineering, Colorado State University, Fort Collins, CO 80523

IEEE Log Number 8933047 plasma generated inside a molybdenum tube $90 \mathrm{~cm}$ in length with an inside diameter of $0.9 \mathrm{~cm}$ by the excitation produced by two glow discharge electron guns. A compact tantalum ribbon heater surrounds the plasma tube and produces the necessary temperature to vaporize silver and obtain the metal vapor density required for laser operation. The plasma tube and the heater are placed on the axis of an electromagnet that produces a magnetic field with the purpose of guiding and confining the electron beam. The magnetic field minimizes the scattering of energetic beam electrons to the walls of the plasma tube and consequently allows for a more efficient deposition of the electron beam energy into the plasma. An electron beam is injected into the plasma tube from each end using two electron guns connected to the same dc power supply through separate ballast resistors. The use of a pair of electron guns instead of one allows for the generation of two counterpropagating electron beams which produce a more uniform plasma and increases the available pumping power. The electron guns, which have a $0.5 \mathrm{~cm}$ diameter hole through their axis to allow the passage of laser radiation, are constructed using molybdenum-magnesium oxide sintered cathodes that can produce $1 \mathrm{~A} \mathrm{dc}$ electron beams in an oxygen free atmosphere [8], [9]. The beam electrons are emitted following ion bombardment of a cathode area that is $2.6 \mathrm{~cm}$ in diameter.

A small neon flow is maintained through the electron gun chambers which are placed at each end of the plasma tube. Neon is introduced through two independently controlled needle valves and is circulated by a rotary pump. A small pressure difference ( 0.1 torr) is maintained between both chambers to establish a small gas flow through the plasma tube with the purpose of obtaining a more even distribution of silver vapor in the entire medium. For all practical purposes, the silver vapor is confined to the inside of the plasma tube, and the electron guns which are placed $16 \mathrm{~cm}$ from the ends of the plasma tube operate in a pure neon atmosphere.

The current-voltage characteristics of the high-voltage neon glow discharge that produces the electron beams differ from those for the helium discharges used in previous experiments. This is a consequence of the difference in the ionization cross section, ionization energy and ionic mobility of both gases [10]. The $I-V$ characteristics of the high-voltage neon glow discharge are illustrated in Fig. 1. The data were obtained operating the two electron guns simultaneously in the presence of a magnetic field as is done for laser operation. The 


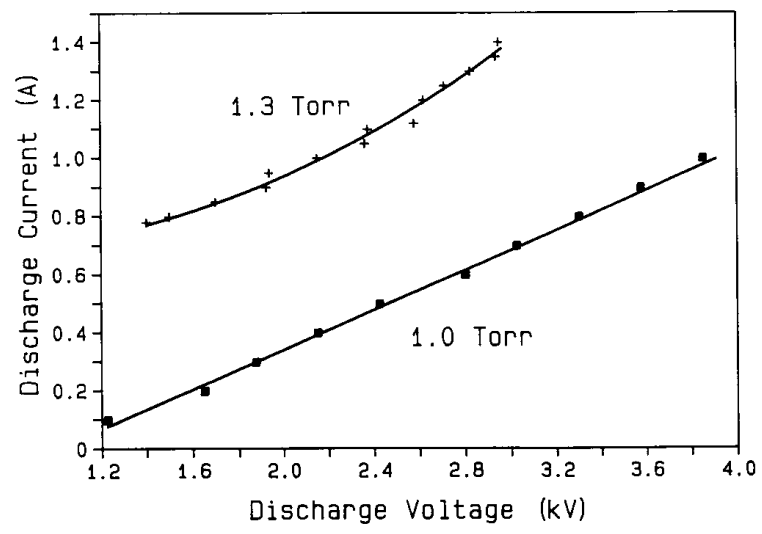

Fig. 1. Total discharge current versus voltage of the glow discharge produced by two electron guns connected in parallel to the same power supply in a neon atmosphere at a pressure of 1.0 and 1.3 torr. Active area of the molybdenum-magnesium oxide cathodes are $2.6 \mathrm{~cm}$ in diameter.

electromagnet current was adjusted to produce a magnetic field of $3.07 \mathrm{kG}$ in the center of the plasma tube. At $16 \mathrm{~cm}$ from the ends of the plasma tube, where the emitting surfaces of the electron guns are located, the corresponding value of the magnetic field was measured to be 27 Gauss.

Fig. 2(a) illustrates the variation of the laser output power of the $478.8 \mathrm{~nm}$ line of AgII as a function of the discharge current. The data correspond to the use of a nonoptimized optical cavity constructed with a flat total reflecting mirror and a $5 \mathrm{~m}$ radius of curvature output coupling mirror having a transmissivity of 1 percent at $478 \mathrm{~nm}$. The discharge current threshold for laser action occurred at $0.78 \mathrm{~A}$, and the $\mathrm{CW}$ output power of the blue line increased linearly to a value of 60 $\mathrm{mW}$ at the maximum electron beam discharge current investigated, $1.15 \mathrm{~A}$. The heater voltage and current were $64 \mathrm{~V}$ and $131 \mathrm{~A}$, respectively, and the value of the magnetic field was $2.9 \mathrm{kG}$. Due to the higher ionization cross section of neon and the corresponding shorter electron mean-free path for the beam electrons, the operation of the $\mathrm{Ne}-\mathrm{Ag}$ laser requires lower pressure than the $\mathrm{He}-\mathrm{Ag}$ infrared laser [2]. The optimum average neon pressure was found to be 1.3 torr. We note that with the optical cavity and electron gun geometry described above, only approximately 20 percent of the total plasma volume is used to generate laser radiation, and an increased laser output power could be obtained by optimizing the overlap between the mode volume and the electron beam plasma volume. The variation of the laser output power as a function of the externally applied magnetic field is illustrated in Fig. 2(b). The dependence is similar to that previously observed for the $840.4 \mathrm{~nm}$ line of AgII in a He-Ag mixture [2]. In both cases, the laser output intensity increases as a function of the axial magnetic field as a consequence of a better confinement of the beam electrons and reaches a maximum at approximately $2.7 \mathrm{kG}$. A further increase in the magnetic field causes the laser output power to decrease.

When a cavity was implemented using two high reflecting ultraviolet mirrors, $\mathrm{CW}$ laser action was readily obtained in the $318.1 \mathrm{~nm}$ line of AgII. Threshold for laser oscillation occurred at $0.55 \mathrm{~A}$, and the laser output power increased

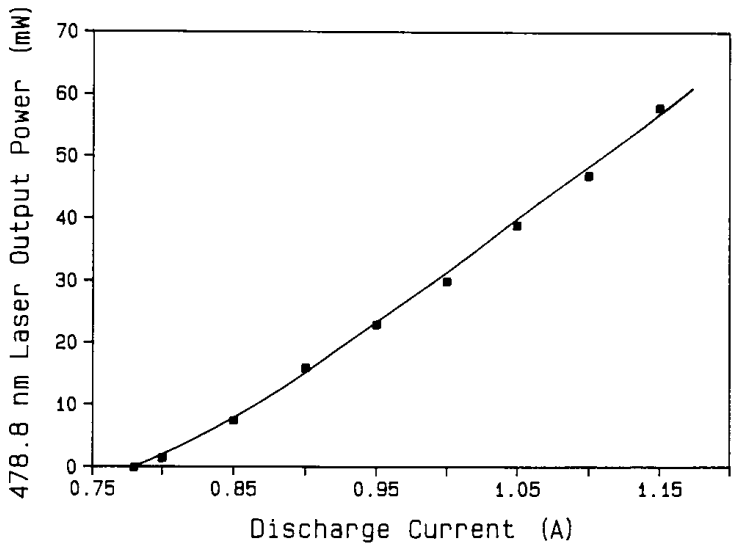

(a)

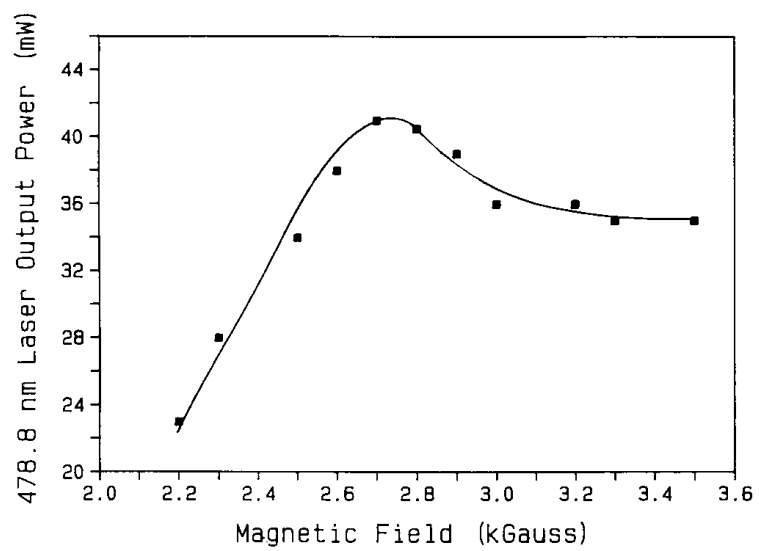

(b)

Fig. 2. Laser output power of the $478.8 \mathrm{~nm}$ line of Agll as a function of: (a) electron beam discharge current for a magnetic field of $2.9 \mathrm{kG}$, (b) externally applied magnetic field of a discharge current of $1.1 \mathrm{~A}$. The neon pressure was 1.3 torr.

linearly without signs of saturation up to the maximum electron beam discharge current investigated, 1.0 A. For comparison, when high reflectivity mirrors in the blue were used, the current threshold for laser oscillation in the $478.8 \mathrm{~nm}$ line was measured to be similar, $0.44 \mathrm{~A}$. By substituting one of the high reflecting ultraviolet mirrors with an output coupler having a transmissivity of 1.3 percent at $318 \mathrm{~nm}$ and a radius of curvature of $10 \mathrm{~m}$, a maximum $\mathrm{CW}$ laser output power of $14 \mathrm{~mW}$ was measured. The dependence of the ultraviolet laser power as a function of electron beam discharge current is shown in Fig. 3 for a neon pressure of 1.3 torr. For electron beam discharge currents between $1.2 \mathrm{~A}$ and the maximum investigated, $1.45 \mathrm{~A}$, the laser output power displays a linear increase with the current. This is a consequence of a similar rate of increase in the creation of ground state neon ions by electron impact ionization. When the neon pressure was lowered to 1 torr the current threshold for ultraviolet laser oscillation decreased to $0.68 \mathrm{~A}$, but the maximum laser power obtained at this pressure was only $5 \mathrm{~mW}$.

In summary, we have demonstrated the operation of an electron beam excited $\mathrm{CW}$ laser in the ultraviolet for the first time, and we have expanded the number of possible laser lines 


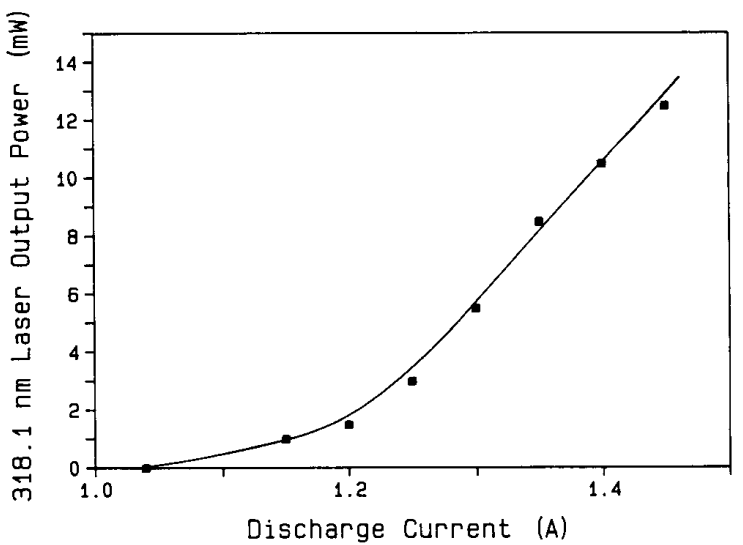

Fig. 3. Variation of the laser output power of the $318.1 \mathrm{~nm}$ line of AgII as a function of electron beam discharge current. The external magnetic fielc was $2.9 \mathrm{kG}$, and the neon pressure was 1.3 torr. The heater voltage and current were $62 \mathrm{~V}$ and $135 \mathrm{~A}$, respectively. The high reflecting mirror had a $15 \mathrm{~m}$ radius of curvature and the output coupling mirror had a $10 \mathrm{~m}$ radius of curvature with a transmissivity of 1.3 percent.

for this type of excitation by demonstrating the use of neon as a buffer gas.

\section{ACKNOWLEDGMENT}

The authors want to thank K. Lewis, T. Prabhuram, M. Villagran, and F. Gonzalez for their assistance in the construction and initial testing of the laser device. We also want to thank L. Spinelli and T. Johnston of Coherent Inc. for lending us the mirrors used in these experiments and W. Silfvast for encouraging discussions. H. L. Mancini acknowledges the support of CONICET, Argentina.

\section{REFERENCES}

[1] J. J. Rocca, J. D. Meyer, and G. J. Collins, "1-W CW Zn ion laser," Appl. Phys. Lett., vol. 43, pp. 37-39, 1983.

[2] B. Wernsman, T. Prabhuram, K. Lewis, F. Gonzalez, M. Villagran and J. J. Rocca, "CW silver ion laser with electron beam excitation," IEEE J. Quantum Electron., vol. QE-24, pp. 1554-1556, 1988.

[3] J. J. Rocca, H. L. Mancini, and B. Wernsman, "Cd recombination laser in a plasma generated by an electron beam," IEEE J. Quantum Electron., vol. QE-22, pp. 509-512, 1986.

[4] J. J. Rocca, J. D. Meyer, Z. Yu, M. Farrell, and G. J. Collins, "Multikilowatt electron beams for pumping $\mathrm{CW}$ ion lasers," Appl. Phys. Lett., vol. 41, pp. 811-813, 1982.

[5] J. J. Rocca, J. D. Meyer, B. G. Philstrom, and G. J. Collins, "CW laser action in atomic fluorine," IEEE J. Quantum Electron., vol. QE-20, pp. 625-628, 1984

[6] W. R. Bennett, Jr., Atomic Gas Laser Transition Data, A Critical Evaluation. New York: IFI Plenum, 1980.

[7] J. R. McNeil, W. L. Johnson, G. J. Collins, and K. B. Persson, "Ultraviolet laser action in He-Ag and Ne-Ag mixtures," Appl. Phys. Lett., vol. 29, pp. 172-174, 1976. See also R. Solanki, W. M. Fairbank, Jr., and G. J. Collins, "Multiwatt operation of CuII and AgIl hollow cathode lasers," IEEE J. Quantum Electron., vol. QE-16, pp 1292-1294, 1980

[8] J. J. Rocca, J. D. Meyer, M. R. Farrell, and G. J. Collins, "Glowdischarge-created electron beams: Cathode materials, electron gun designs, and technological applications," J. Appl. Phys., vol. 56, pp. 790-797, 1984

[9] B. Szapiro and J. J. Rocca, "Electron emission from glow-discharge cathode materials due to neon and argon ion bombardment," J. Appl. Phys., vol. 65, pp. 3713-3715, 1989.

[10] E. W. McDaniel, Collisional Phenomena in Ionized Gases. New York: Wiley, 1964. 